\title{
Comparative study of conventional US, contrast enhanced US and enhanced MR for the follow-up of prostatic radiofrequency ablation
}

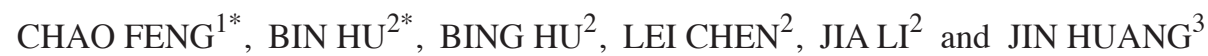 \\ Departments of ${ }^{1}$ Urology, ${ }^{2}$ Ultrasound in Medicine and ${ }^{3}$ Pathology, Shanghai Institute of Ultrasound in Medicine, \\ Shanghai Jiaotong University Affiliated Sixth People's Hospital, Shanghai 200233, P.R. China
}

Received August 18, 2015; Accepted January 6, 2017

DOI: $10.3892 /$ etm.2017.4399

\begin{abstract}
The aim of the present study was to evaluate and compare the effectiveness of different imaging methods during follow-up of prostatic radiofrequency ablation. Prostatic radiofrequency ablation (RFA) was performed in 20 healthy beagle dogs. Various imaging examinations were used to monitor the results of RFA, including conventional ultrasound (US), contrast enhanced ultrasound (CEUS) and enhanced magnetic resonance (MR). Imaging exams were performed at five phases: Immediately following RFA, one week later, one month later, three months later and six months later. The morphology for each imaging test and histological results were recorded and compared in each phase. Based on the actual results from autopsy, the accuracy of those imaging exams was evaluated. The canine prostate gland demonstrated typical coagulative necrosis immediately following RFA. The lesion would develop into stable cyst if no other complications occurred within the six-month follow-up. Regarding the RFA lesion volume measurement and the reflection of pathological changes, conventional US was not able to accurately measure the volume of RFA lesion and missed many more details concerning the RFA-treated area than CEUS and MR during the three months. The results from CEUS exhibited comparable accuracy to those from enhanced MR at each phase. However, there were no significant differences in the results from US, CEUS and MR at six months, which may contribute to the complete formation of lesion cyst. In the early phase, conventional US was not sufficient for evaluating the efficacy
\end{abstract}

Correspondence to: Dr Bin $\mathrm{Hu}$, Department of Ultrasound in Medicine, Shanghai Institute of Ultrasound in Medicine, Shanghai Jiaotong University Affiliated Sixth People's Hospital, 600 Yi Shan Road, Shanghai 200233, P.R. China

E-mail: fengchao9901790@aliyun.com

\section{${ }^{*}$ Contributed equally}

Key words: prostate, radiofrequency ablation, ultrasound, magnetic resonance imaging of RFA. Enhanced US and MR provided clear images and accurate information. However, CEUS has the advantage of being more economical, using more convenient equipment and faster scanning, thus identifying it as the more feasible choice. Furthermore, no notable advantages were observed among any image examinations in the long-term follow-up.

\section{Introduction}

The worldwide incidence of prostate cancer has been rising in recent years and prostate cancer has become a major threat for aged male populations. Each year, 914,000 new cases are diagnosed, and 258,000 cases lead to mortality (1). At present, radical prostatectomy is the standard treatment for localized prostate cancer; however, this surgical procedure may have a notable negative impact on patients, such as incontinence or erectile dysfunction (2). Radiofrequency ablation (RFA) is gaining popularity as a method of treating prostate diseases, as it is an interventional therapy that causes less trauma, quicker recovery and fewer complications (3).

In order to obtain results from RFA, the use of a real-time prostatic imaging monitor is necessary. At present, conventional transrectal ultrasound (US) is typically used for guiding prostate RFA, allowing the procedure to be visualized in real time. However, the indication of RFA is vague and the accuracy of imaging results is not reliable $(3,4)$. In addition to conventional US, contrast enhanced US (CEUS) and enhanced magnetic resonance (MR) have also been considered to be valuable tools in the follow-up monitoring of RFA (5-8). In previous studies, the feasibility of using contrast enhanced US and enhanced MR in evaluating the results of liver RFA has been investigated (9-11). In a previous study by the current authors, CEUS was used to evaluate the short-term results of prostate RFA (12). However, the long-term value of CEUS and enhanced MR in monitoring the results of RFA remains to be determined. To the best of our knowledge, there has been no research comparing the efficacy of these imaging methods in the dynamic observation of prostate RFA therapy. Therefore, in the present study, different imaging methods were used to dynamically track changes in RFA lesions in a beagle animal model in order to identify the optimal imaging exam for the clinical observation of RFA lesions in prostate cancer. 


\section{Materials and methods}

Animal model. A total of 20 healthy male beagle dogs were purchased from the Experimental Animal Institution, Shanghai Jiaotong University affiliated Sixth People's Hospital (Shanghai, China). The mean age was $5.32 \pm 1.21$ years, and mean body weight was $17.4 \pm 3.32 \mathrm{~kg}$. The current protocol was approved by the Institutional Animal Use and Care Committee of Shanghai Jiatong University Affiliated Sixth People's Hospital. Dogs were sedated and anesthetized prior to experiments with sodium pentobarbital (30 mg/kg; Sinopharm Chemical Reagent Co., Ltd., Shanghai, China). Cardiac and respiratory parameters were monitored throughout the procedures. An intravenous fluid channel was established through an 18-gauge angiocatheter (BD Biosciences, Franklin Lakes, NJ, USA) placed in a forelimb vein.

Surgical procedure. The surgical procedure was performed as previously described (12). Briefly, all beagle dogs underwent prostatic RFA treatment. RFA was performed with a Model 1,500 generator, using a StarBurst SDE probe (RITA Medical Systems, Inc., Mountain View, CA, USA) to create a lesion. Briefly, a Foley catheter was inserted to cool the urethra via irrigation with saline. A three-hook active needle was subsequently inserted into the prostate through the anterior wall of the rectum under transrectal ultrasonography (TRUS) guidance. The orientation of the needle was just parallel to the ultrasonography probe during the insertion. A lesion was created in the right and left lateral lobes of the prostate. RF energy $(250 \mathrm{~W})$ was delivered for $5 \mathrm{~min}$ at a target temperature of $90^{\circ} \mathrm{C}$. Surgery on all dogs was performed by an experienced technician under anesthesia, as previously described. Each dog was intramuscularly administered $80 \mathrm{mg} / \mathrm{kg}$ gentamycin (Sinopharm Chemical Reagent Co., Ltd.) for one week following the end of surgery.

CEUS. Sonovue (Bracco, Milan, Italy) was selected as a contrast agent for the present study. The agent was infused in boluses of $2.4 \mathrm{ml}$ followed by $5 \mathrm{ml}$ physiological saline immediately prior to imaging. A dynamic TRUS was performed using a Technos MPX DU8 scanner (Esaoate SpA, Genova, Italy) with an EC123 end-fire probe.

MR imaging (MRI) examination. MRI examination was performed in beagle dogs under general anesthesia. Dogs were placed in the prone position within the gantry of a clinical 1.5 T MRI system (SIGNA; GE Healthcare Bio-Sciences, Pittsburgh, PA USA). MRI was performed using fast spin-echo $\mathrm{T} 1$ weighted image (WI), T2W1 and short T1 inversion recovery sequences. The dynamic enhanced sequence was performed $5 \mathrm{sec}$ following intravenous administration of gadopentetic acid $(0.1 \mathrm{mmol} / \mathrm{kg}$; Merck KGaA, Darmstadt, Germany). The imaging protocol of enhanced MRI was as follows: Repetition time, $150 \mathrm{msec}$; echo time, $1.9 \mathrm{msec}$; matrix size, 256x160; slice thickness, $3 \mathrm{~mm}$; field of view $18 \times 13 \mathrm{~mm}$.

Protocol of follow-up. All 20 dogs were randomly assigned into 5 groups $(n=4)$. Dogs in group 1 were sacrificed via administration of sodium pentobarbital $(120 \mathrm{mg} / \mathrm{kg})$ immediately after RFA following imaging exams. Dogs in groups
2 to 5 were executed at one week, one month, three months and six months following RFA, respectively. At each time point, prior to sacrifice, every living dog received conventional TRUS, dynamic CEUS exam and enhanced MRI of their prostate glands. Three regular doctors, including one urologist, one radiologist and one ultrasonic doctor, subsequently evaluated the images from those exams with Sante Dicom Viewer software (version 8.1.3; Santesoft, Ltd., Athens, Greece). Following the examinations at each time point, dogs in the appropriate group were sacrificed prior to pathological examinations.

Pathological assessment. A single pathologist who was blinded to the graphic measurements of the RFA lesions performed all histopathological analyses. Prostate tissue was harvested and the ventral surface of the prostate was sectioned in sequential $4 \mathrm{~mm}$ slices from the apex to the base along the transverse plane. Slices were soaked in $1 \%$ triphenyltetrazolium chloride (Sinopharm Chemical Reagent Co., Ltd.) for 5-10 min at room temperature to distinguish between viable and nonviable cells and clearly indicate thermal coagulation. The maximal diameters in the transverse and sagittal directions were measured with a ruler and the height was calculated by adding the thickness of the affected tissue slices. Following measurement, routine histology with hematoxylin and eosin stain for light microscopy was performed. Briefly, the slices were dewaxed under xylene. The dehydration process was performed under ethanol (concentration, 60-100\%) and slices were stained with henatoxylin and eosin, subsequently. Images were analyzed using Image J software (version 4.17; National Institutes of Health, Bethesda, MD, USA).

Statistical analysis. Thermal lesion volume was calculated according to the expected ellipsoid shape using the following equation: Volume $=1 / 6 x \pi x$ (axbxc), where $a, b$ and $c$ are the maximal radius in the transverse, anterior-posterior, and craniocaudal directions, respectively. All measured volumes are expressed as the mean \pm standard deviation in $\mathrm{cm}^{3}$. The Wilcoxon matched pair rank test was used to evaluate any systematic differences between sonography or MRI imaging and pathology measurements using SPSS 11.0 software (SPSS, Inc., Chicago, IL, USA).

\section{Results}

24 h after RFA. Gross examination indicated that the lesion site exhibited a round or elliptical shape and brownish color, with a red hemorrhage ring at the rim (Fig. 1A). Histological examination revealed a clear change from the necrotic zone to the normal gland (Fig. 1B). Using conventional US, the RFA lesion demonstrated the inhomogeneous echo region, with no distinguishable border between the lesion and normal tissue. In some specimens, the hypoechoic dot, induced by the probe, was detected in the center of the lesion (Fig. 1C). However, under CEUS, the RFA lesion appeared clear, as the agent was not able to perfuse into the lesion. Therefore, the border between the lesion and normal tissue was distinguishable by CEUS. (Fig. 1D). Additionally, enhanced MRI also presented the lesion site clearly and a clear border circus was also visible in this examination (Fig. 1E; Table I). 
Table I. Comparison of the lesion volume measured via regular conventional US, CEUS and MRI at each time point.

Lesion volume, $\mathrm{cm}^{3}$ (mean \pm standard deviation)

\begin{tabular}{lcccc}
\cline { 2 - 4 } Time point & Conventional US & CEUS & Enhanced MRI & Gross \\
\hline $24 \mathrm{~h}$ & $1.86 \pm 0.53^{\mathrm{a}}$ & $1.27 \pm 0.51$ & $1.23 \pm 0.50$ & $1.27 \pm 0.52$ \\
1 week & $1.08 \pm 0.06^{\mathrm{a}}$ & $1.53 \pm 0.09$ & $1.56 \pm 0.11$ & $1.54 \pm 0.10$ \\
1 month & $0.53 \pm 0.15$ & $0.55 \pm 0.15$ & $0.58 \pm 0.12$ & $0.55 \pm 0.13$ \\
3 months & $0.75 \pm 0.63$ & $0.78 \pm 0.71$ & $0.82 \pm 0.72$ & $0.81 \pm 0.74$ \\
6 months & $0.53 \pm 0.56$ & $0.41 \pm 0.41$ & $0.46 \pm 0.47$ & $0.40 \pm 0.39$ \\
\hline
\end{tabular}

${ }^{\mathrm{a}} \mathrm{P}<0.05$ vs. gross exam; US, ultrasound; CE, contrast enhanced; MRI, magnetic resonance imaging.
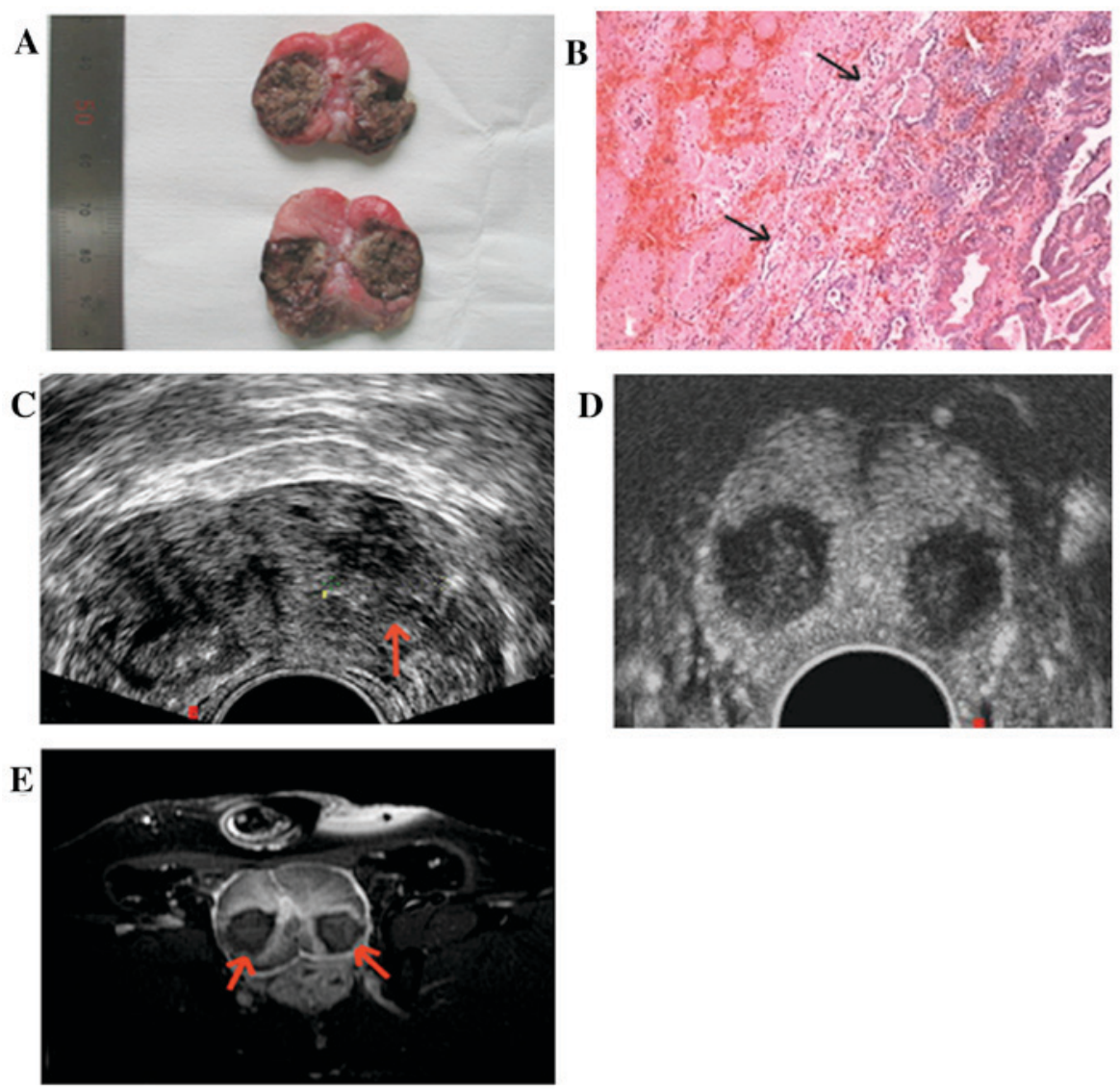

Figure 1. The graphic and histological results of RFA after $24 \mathrm{~h}$. (A) Gross view of prostate. (B) Hematoxylin and eosin imaging of RFA lesion. Arrows indicate the necrotic zone. (C) Conventional US. Arrow indicate the inhomogeneous echo region. (D) Contrast enhanced US. (E) Enhanced magnetic resonance imaging (T1 weighted image sequence). Arrows indicate the clear lesion site. Original magnification of images, x100. RFA, radiofrequency ablation; US, ultrasound.

One week after RFA. In gross view, many lesions exhibited irregular cavities with a dark brown liquid inside, which was considered as the necrotic tissue and hemorrhage. Dark brown hemorrhage rings were also observed at the rim (Fig. 2A). In the histological examination, edema and hemorrhage were observed surrounding the necrotic tissue with a marked inflammatory reaction. Granulation was also observed in the tissue surrounding the lesion, which was evoked by the inflammatory reaction (Fig. 2B). Conventional US presented the lesion as a hypoechoic cyst with inhomogeneous echo inside; however it exhibited a marked annular blood flow signal around it. At this time ultrasound was still unable to define the lesion border (Fig. 2C). Following enhancement, the lesion exhibited no enhancement and an annular enhancement was visible around it. The border of the lesion was distinguishable (Fig. 2D). The lesion was also clear with enhanced MRI. However, the high signal ring surrounding the lesion was markedly obvious in this phase compared with group 1 (Fig. 2E; Table I).

One month after RFA. At this phase, the lesion still exhibited the solid structure with irregular necrosis. The hemorrhage 

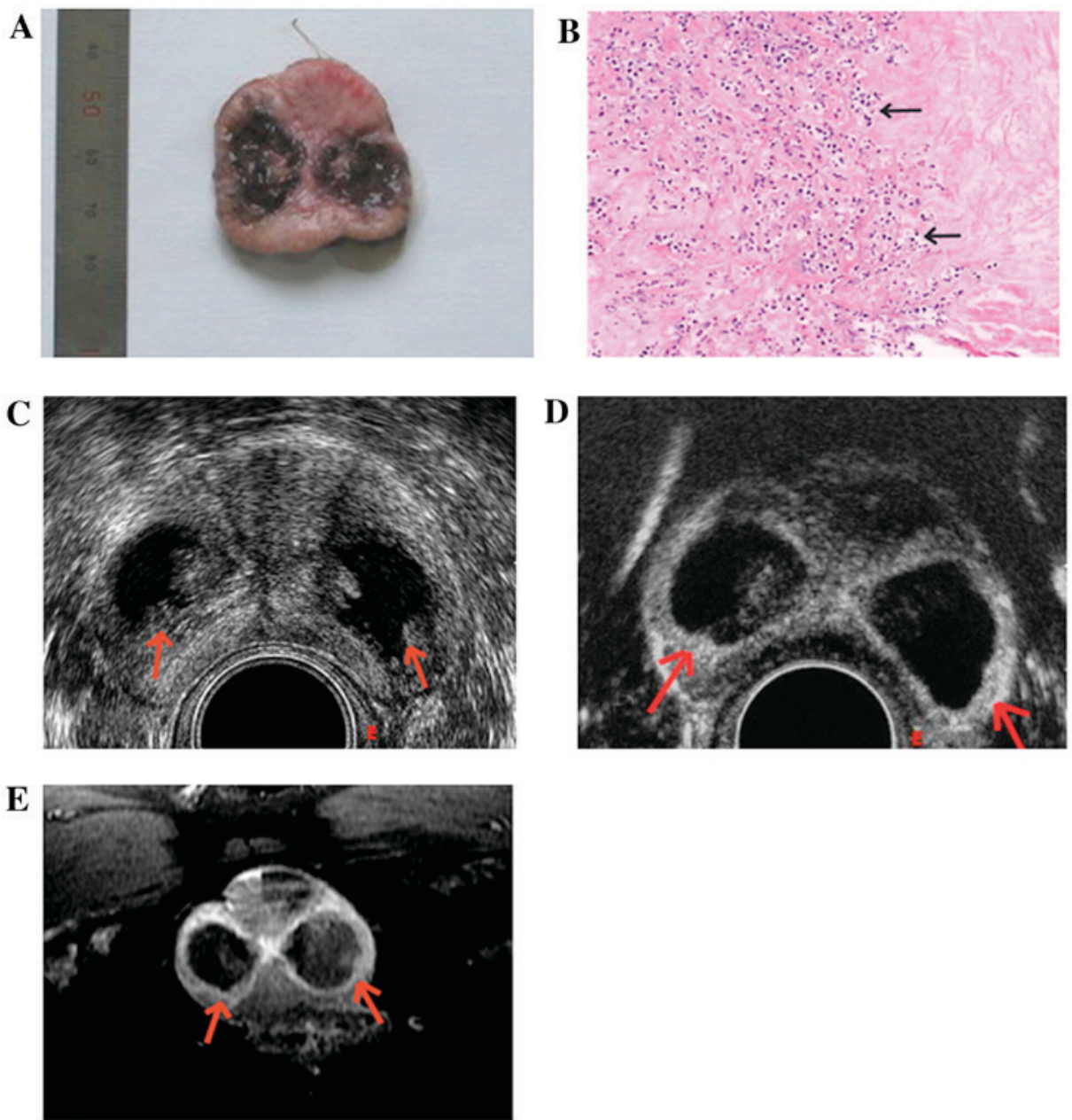

Figure 2. The graphic and histological results of RFA after one week. (A) Gross view of prostate. (B) Hematoxylin and eosin imaging of RFA lesion. Arrows indicate the hemorrhage rim. (C) Conventional US. Arrows indicate the hypoechoic cyst. (D) Contrast enhanced US. Arrows indicate the hypoechoic cyst without the enhancement. (E) Enhanced magnetic resonance imaging (T1 weighted image sequence). Arrows indicate the high signal ring surrounding the lesion. Original magnification of images, x100. RFA, radiofrequency ablation; US, ultrasound.

rim was not detected in the autopsy sample. The lesion was surrounded by white fibrous tissue. In some specimens, a small cyst was visible within the lesion. The cyst had a clear border and smooth cystic wall (Fig. 3A). The pathological change of the lesion exhibited some diversity. Some specimens exhibited lesion sites that had developed into cysts with a yellow-green liquid inside (Fig. 3B). Some exhibited coarse walls and some had smooth walls. Some lesions had an irregular greyish yellow appearance with no clear boundary. US examination detected that some lesion appeared as hypoechoic, irregular areas with uneven echo signals inside and no clear border. Some of these lesions exhibited small, irregular cysts inside. These lesions had rich blood flow inside and short radial blood flow outside (Fig. 3C). The outer part of the lesion exhibited strong early enhancement with CEUS, whereas the inner part showed no such enhancement. Other lesions had the appearance of a regular shaped cyst. The cyst had no blood flow inside but had annular flow signal around the cyst wall. In CEUS, the tissue adjacent to the cyst wall showed early enhancement; however the cyst exhibited no enhancement (Fig. 3D). For subjects with no cyst formation, enhanced MRI was able to clearly define its border and detect surrounding annular enhancement (Fig. 3E). For subjects with cyst formation, MRI showed a typical cyst with clear border, and enhanced MRI showed annular enhancement of cyst wall.

Three months after RFA. Similar to the group observed at one month, the RFA lesions exhibited structural changes to the cyst, which had clear light green liquid inside (Fig. 4A). Apart from one specimen that developed an abscess, other specimens exhibited a markedly reduced inflammatory reaction in the histological section. Cystic walls were covered with an intact glandular epithelium (Fig. 4B). US exhibited some lesions as irregular cysts with smooth walls and no echo inside and cysts exhibited a surrounding annular blood flow (Fig. 4C). CEUS detected that cysts had surrounding annular enhancement. However, the effect of enhancement in this phase was weaker than in all earlier stages (Fig. 4D). In the MRI examination, the enhancement imaging still detected a high signal rim around the cyst. It also detected the border line between normal tissue and the lesion (Fig. 4E; Table I).

Six months after RFA. At this phase, all specimens exhibited similar changes. Lesion sites all appeared as oval-shaped cysts with thin, smooth walls and clear light yellow fluid, surrounded by white fibrous tissue (Fig. 5A). Histological analysis revealed 
A

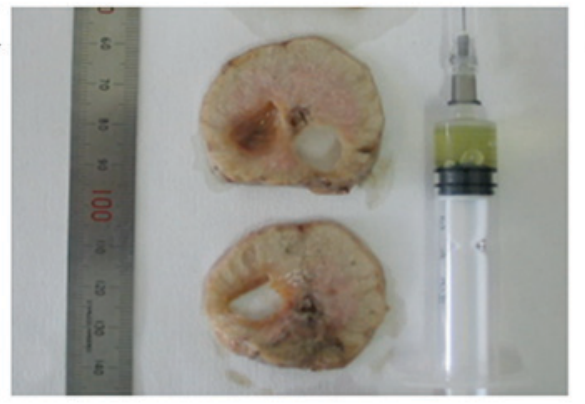

C

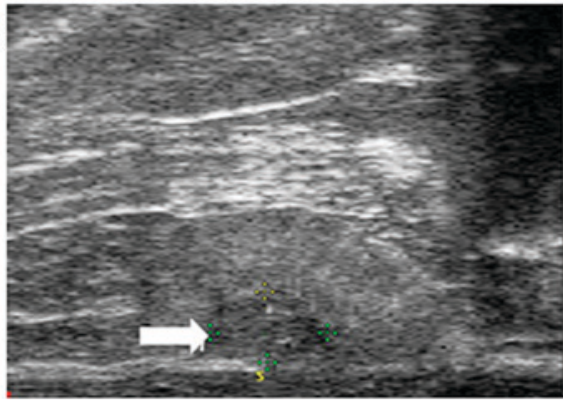

E

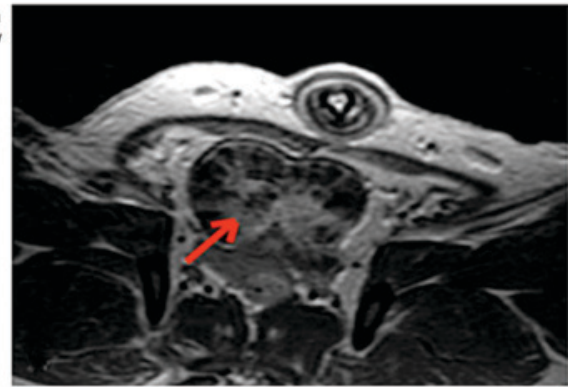

B

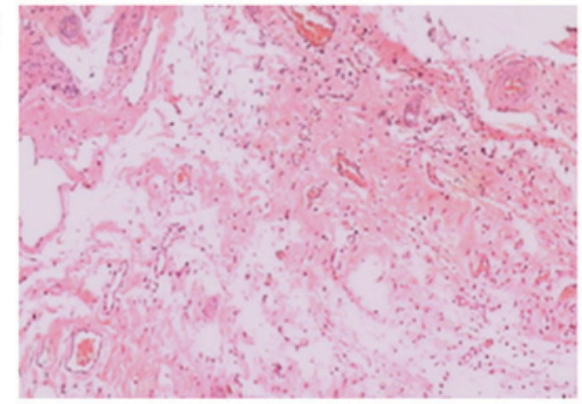

D

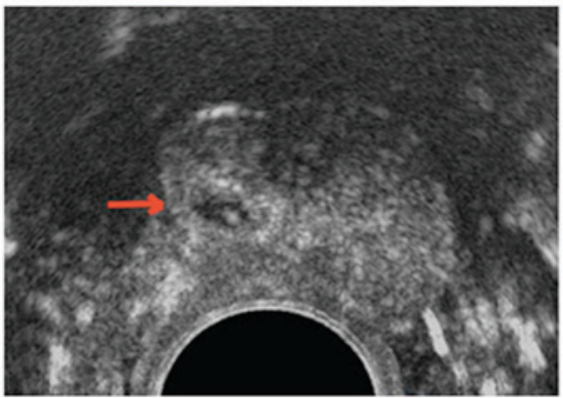

Figure 3. The graphic and histological results of RFA after one month. (A) Gross view of prostate. (B) Hematoxylin and eosin imaging of RFA lesion. (C) Conventional US. Arrows indicate the hypoechoic, irregular areas with uneven echo signals inside. (D) Contrast enhanced US. Arrows indicate the tissue adjacent to the cyst wall showed early enhancement. (E) Enhanced magnetic resonance imaging (T1 weighted image sequence). Arrows indicate the clearly defined cyst border and detect surrounding annular enhancement. Original magnification of images, x100. RFA, radiofrequency ablation; US, ultrasound.

that the wall was covered with simple columnar epithelium with local squamous metaplasia (Fig. 5B). Conventional US demonstrated that all lesions had developed into oval-shaped cysts with clear boundaries and a transparent no echo zone inside. The cysts had smooth walls with linear high echo and traces of blood flow surrounding the cyst wall (Fig. 5C). The cyst, cyst wall and surrounding tissue all exhibited no marked enhancement in CEUS (Fig. 5D). MRI detected typical cysts and wall with weaker enhancement than indicated in previous tests (Fig. 5E; Table I).

\section{Discussion}

Radiofrequency ablation is a minimally invasive therapy, which is suitable for tumors that are difficult to reach with surgical approaches. It has been successfully applied in hepatic cancer and lung cancer with satisfactory results $(4,13)$. The RFA of prostate cancer is also considered as a promising complement for surgery (3). In previous studies, the effectiveness and value of RFA in localized prostate cancer has been investigated $(3,14)$. Although the results of initial studies were encouraging, various limitations still exist regarding this procedure. Similar to its application in other types of cancer, the primary issue with RFA in solid tumors such as liver carcinoma, renal carcinoma and prostatic tumor, is the high rate of recurrence $(14,15)$; therefore, follow-up after RFA is of great importance. Conventional US is always used when performing RFA; however, various studies agree that it is unable provide accurate information about the lesion during follow-up due to the limitations of this technique $(3,4)$. At present, various graphic examinations, such as CT and MRI, have been considered to aid in evaluation of RFA $(16,17)$.

Unlike in hepatic or lung tumors, computed tomography (CT) scanning is not very sensitive in prostate cancer (18). It seems that MRI is most suitable for evaluating the prostate as it has the highest sensitivity to soft tissue, therefore, it has been used to evaluate the results of RFA (19). However, this technique has various limitations, due to the larger equipment, higher cost and strict indication. Notably, CEUS functions similarly to enhanced CT or MR as it requires injected contrast media to indicate the distribution of blood flow in different tissues and organs. Furthermore, it has a markedly reduced radiation exposure compared with CT scanning and a lower cost than MRI (18). It also does not require the use of complex equipment and is therefore easy to perform, which may reduce waiting times for patients. These merits support the use of 

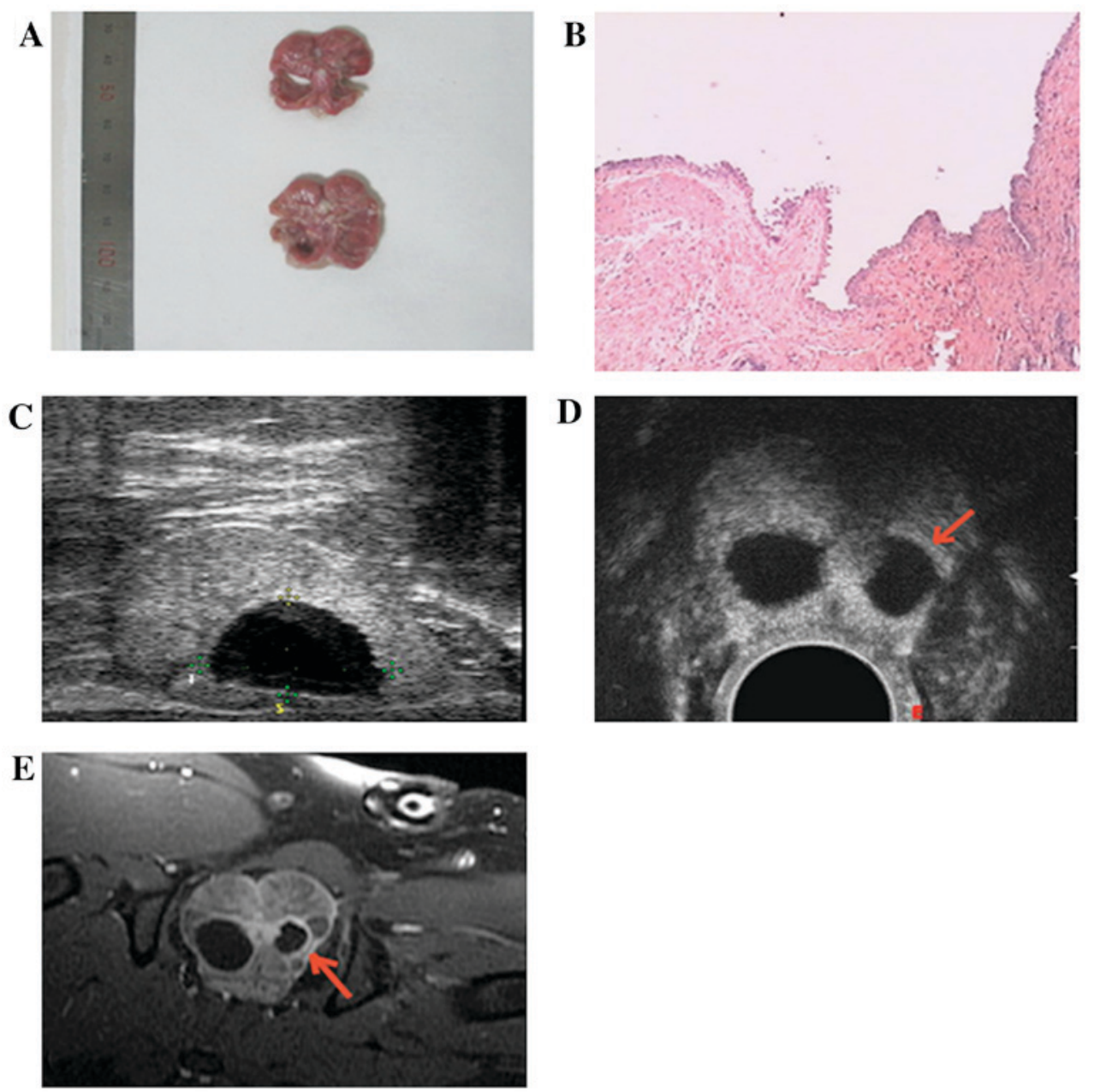

Figure 4. The graphic and histological results of RFA after three months. (A) Gross view of prostate. (B) Hematoxylin and eosin imaging of RFA lesion. (C) Conventional US. (D) Contrast enhanced US. Arrows indicate the surrounding annular enhancement of cysts. (E) Enhanced magnetic resonance imaging (T1 weighted image sequence). Arrows indicate high signal rim around the cyst. Original magnification of images, x100. RFA, radiofrequency ablation; US, ultrasound.

CEUS as the most effective method of monitoring changes in the prostate following RFA.

The beagle dog was chosen as the animal model in the present study for a number of reasons. Firstly, the anatomy of the prostate in dogs is similar to that in humans, meaning that it can more accurately mimic prostate cancer in humans. Secondly, performing MRI and CEUS examination in a larger animal is more similar to actual clinical practice. Finally, it is easier for the investigator to manipulate MRI and CEUS in larger animals than in small animals.

The short-term value of prostate RFA has been determined previously (12). As the long-term imaging of changes in prostatic RFA lesion has not yet been reported, the present study was performed to identify long-term morphological and imaging changes in prostatic RFA lesions by replicating the long-term follow-up, and to identify the optimal monitoring method to be used during this follow-up period.

In the pathological study, affected tissue was observed to undergo co-agulative necrosis $(20,21)$ following RFA. Following the absorption or expulsion of necrotic tissue, the RFA lesion developed into a cyst with an epithelial lining and scar tissue wall. Foster et al previously reported pathological changes in the canine prostate following high-intensity focused
US and the results were largely similar to those of the current study (22). Within three months following RFA, coagulative necrosis coincided with the small cyst. At this phase, the merit of using CEUS and enhanced MRI is clear. Conventional ultrasound was unable to identify necrotic and normal tissue accurately, which was in accordance with previous reports $(19,20)$. The RFA region was also easily detected via CEUS, as the microbubble exhibited a reduced perfusion into the RFA zone. A high signal rim was also observed with enhanced MRI $(18,23)$, which aided the physician in defining the RFA regional signal. Due to the presence of the high signal rim, it seemed that the image quality in enhanced MRI was slightly improved within $24 \mathrm{~h}$ following RFA, compared with CEUS. This rim made it possible to identify the hyperemia and edema zone surrounding the RFA lesion. In this phase, it was difficult for the microbubble to enter this zone. This may explain why a similar zone was not detected by CEUS. Within one week following RFA, the rim was used to identify the edema, hemorrhage and inflammatory response zone surrounding the RFA lesion. In the CEUS examination, the microbubble agent perfused this zone with ease. Therefore, enhanced MRI and CEUS were able to clearly detect the rim. After one month, a dense fibrotic ring formed around the 
A
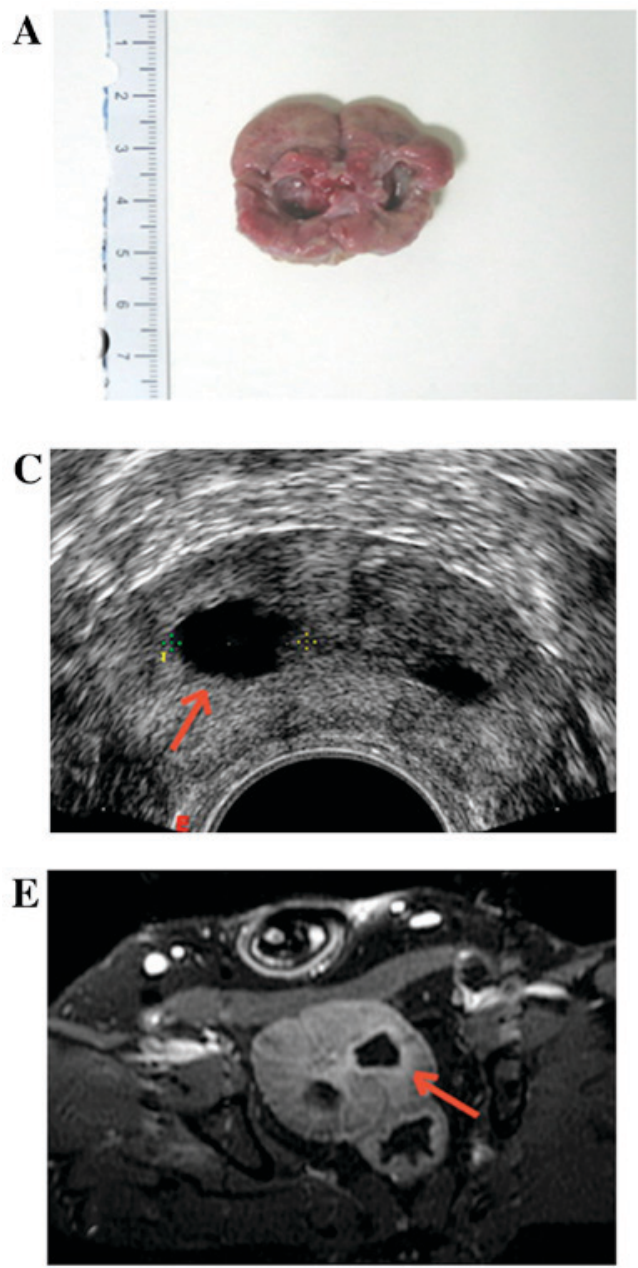

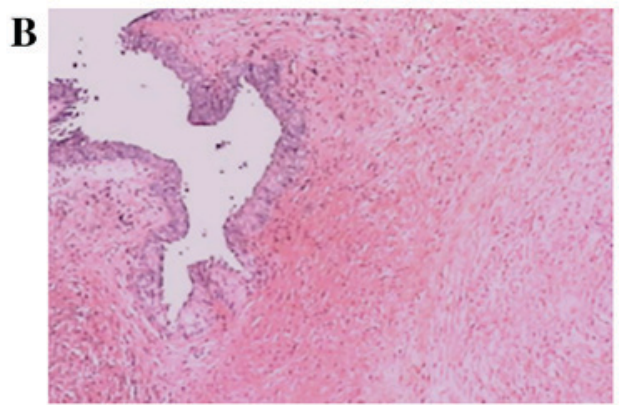

D

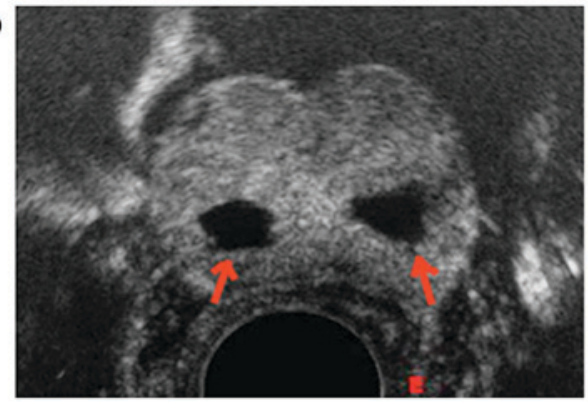

Figure 5. The graphic and histological results after RFA after six months. (A) Gross view of prostate. (B) Hematoxylin and eosin imaging of RFA lesion. (C) Conventional US. Arrows indicate oval-shaped cysts with clear boundaries and a transparent no-echo zone inside. (D) Contrast enhanced US. Arrows indicate the cyst without enhancement (E) Enhanced magnetic resonance imaging (T1 weighted image sequence). Arrow indicates typical cysts and wall with weaker enhancement. Original magnification of images, x100. RFA, radiofrequency ablation; US, ultrasound.

RFA lesion, shrinkage was observed in the edema zone and progressive co-agulative necrosis had occurred. As a result, CEUS and enhanced MRI were able to clearly demonstrate the border. These results suggest that the advantages of enhanced MRI are not applicable to long-term follow-up, during which the image quality of CEUS will be as good as MRI within short period of time.

Three months after RFA, the pathological progression of lesions consisted of absorbing and expelling the necrotic tissue and the RFA lesion had developed into a cyst with an epithelial lining and scar tissue wall. In this phase, conventional US was able to detect the border of the lesion. The accuracy of this measurement was similar to that of enhanced MRI and CEUS, and in some samples, the image quality in CEUS was better than MRI as areas of interest were more easily detected. The continuous shrinkage of edema and inflammatory tissue surrounding the lesion was also apparent. However, it should be noted that the progression of damage repair may vary greatly in different individuals. Throughout the observational period, it was demonstrated that pathological changes did not occur simultaneously in different dogs. However, different specimens may experience different pathological changes at the same time after RFA, whereas two lesions from the same prostate specimen may have different appearances. Stable cysts had formed after one month in some specimens and only occurred at six months in others. At the six-month phase, all lesions had formed cysts. As such, the advantages associated with enhanced MRI and CEUS may be reduced over time.

Compared with US, MRI may be used to view larger areas surrounding the prostate and to clearly show adjacent structures $(18,23)$. As it is a more objective method, the results of MRI are less dependent on the operator. However, MRI requires expensive equipment and a longer scanning time, which limits its clinical application (11). The accuracy of CEUS is similar to that of enhanced MR, whereas its clinical use is cheaper and more convenient than MRI scan (18). Conventional US also has some advantage over MRI, as it may detect subtle structural changes such as the linear high echo of the cyst wall. As such, CEUS may be regarded as an accurate and convenient method of RFA follow-up together with MR.

Although CEUS and enhanced MRI demonstrated merit in the early monitoring of RFA lesions, further studies should investigate alternative monitoring methods, such as diffusion-weighted imaging-MRI.

In conclusion, the current study demonstrated that CEUS and MRI were able to clearly detect the pathological 
progression of RFA lesions in dogs with well-coordinated results of pathological examination. This merit only appears in the early phase after RFA; however, CEUS has the advantage of being faster, cheaper and more convenient, suggesting that its usage may be more promising.

\section{Acknowledgements}

The present study was supported by the Shanghai Sanitation Bureau Project (grant no. 20114167).

\section{References}

1. Siegel R, Ward E, Brawley O and Jemal A: Cancer Statistics, 2011: The impact of eliminating socioeconomic and racial disparities on premature cancer deaths. CA Cancer J Clin 61: 212-236, 2011.

2. De Carlo F, Celestino F, Verri C, Masedu F, Liberati E and Di Stasi SM: Retropubic, laparoscopic, and robot-assisted radical prostatectomy: Surgical, oncological, and functional outcomes: A systematic review. Urol Int 93: 373-383, 2014.

3. Zlotta AR, Djavan B, Matos C, Noel JC, Peny MO, Silverman DE, Marberger M and Schulman CC: Percutaneous transperineal radiofrequency ablation of prostate tumour: Safety, feasibility and pathological effects on human prostate cancer. Br J Urol 81: 265-275, 1998.

4. Wilson SR, Burns PN, Muradali D, Wilson JA and Lai X: Harmonic hepatic US with microbubble contrast agent: Initial experience showing improved characterization of hemangioma, hepatocellular carcinoma, and metastasis. Radiology 215: 153-161, 2000.

5. Rouvière $\mathrm{O}$, Lyonnet $\mathrm{D}$, Raudrant $\mathrm{A}$, Colin-Pangaud $\mathrm{C}$, Chapelon JY, Bouvier R, Dubernard JM and Gelet A: MRI appearance of prostate following transrectal HIFU ablation of localized cancer. Eur Urol 40: 265-274, 2001.

6. Tazaki H, Deguchi N, Baba S, Imai Y and Nakashima J: Magnetic resonance imaging following microwave thermotherapy, laser ablation and transurethral resection in patients with BPH. Urologe A 34: 105-109, 1995.

7. Yokoyama T, Tsugawa M, Nagai A and Kumon H: High-energy transurethral microwave thermotherapy in patients with benign prostatic hyperplasia: Comparative study between 30- and 60-minute single treatments. Acta Med Okayama 58: $151-156,2004$

8. Fullerton GD: Imaging with magnetic resonance. Aip Conf Proc 538: 76-77, 2000.

9. Wu JY, Chen MH, Yang W, Lin SZ, Wu W, Yin SS, Zhang H and Yan K: Role of contrast enhanced ultrasound in radiofrequency ablation of metastatic liver carcinoma. Chin J Cancer Res 24: 44-51, 2012.

10. Bo XW, Xu HX, Wang D, Guo LH, Sun LP, Li XL, Zhao CK, He YP, Liu BJ, Li DD and Zhang K: Fusion imaging of contrast-enhanced ultrasound and contrast-enhanced CT or MRI before radiofrequency ablation for liver cancers. Br J Radiol 89: 20160379, 2016
11. Kamel IR, Bluemke DA, Eng J, Liapi E, Messersmith W, Reyes DK and Geschwind JF: The Role of functional MR imaging in the assessment of tumor response after chemoembolization in patients with hepatocellular carcinoma. J Vasc Interv Radiol 17: 505-512, 2006.

12. Hu B, Hu B, Chen L, Li J and Huang J: Contrast-enhanced ultrasonography evaluation of radiofrequency ablation of the prostate: A canine model. J Endourol 24: 89-93, 2010.

13. Iguchi T, Hiraki T, Gobara H, Fujiwara H, Matsui Y, Soh J, Toyooka S, Kiura K and Kanazawa S: Percutaneous radiofrequency ablation of lung cancer presenting as ground-glass opacity. Cardiovasc Intervent Radiol 38: 409-415, 2015.

14. Djavan B,Zlotta AR, Susani M,Heinz G, Shariat S, Silverman DE, Schulman CC and Marberger M: Transperineal radiofrequency interstitial tumor ablation of the prostate: Correlation of magnetic resonance imaging with histopathologic examination. Urology 50: 986-993, 1997.

15. Kim JH, Yim HJ, Lee KG, Kim SY, Jung ES, Jung YK, Kim JH, Seo YS, Yeon JE, Lee HS, et al: Recurrence rates and factors for recurrence after radiofrequency ablation combined with transarterial chemoembolization for hepatocellular carcinoma: A retrospective cohort study. Hepatol Int 6: 505-510, 2012.

16. Solbiati L, Ierace T, Goldberg SN, Sironi S, Livraghi T, Fiocca R, Servadio G, Rizzatto G, Mueller PR, Del Maschio A and Gazelle GS: Percutaneous US-guided radio-frequency tissue ablation of liver metastases: Treatment and follow-up in 16 patients. Radiology 202: 195-203, 1997.

17. Goldberg SN, Gazelle GS, Compton CC, Mueller PR and Tanabe KK: Treatment of intrahepatic malignancy with radiofrequency ablation: Radiology-pathologic correlation. Cancer 88: 2452-2463, 2000.

18. Hazle JD, Diederich CJ, Kangasniemi M, Price RE, Olsson LE and Stafford RJ: MRI-guided thermal therapy of transplanted tumors in the canine prostate using a directional transurethral ultrasound applicator. J Magn Reson Imaging 15: 409-417, 2002.

19. Guibal A, Bertin C, Egels S, Savier E, Grenier PA and Lucidarme O: Contrast-enhanced ultrasound (CEUS) follow-up after radiofrequency ablation or cryoablation of focal liver lesions: Treated-area patterns and their changes over time. Eur Radiol 23: 1392-1400, 2013.

20. Djavan B, Susani M, Shariat S, Zlotta AR, Silverman DE, Schulman CC and Marberger M: Transperineal radiofrequency interstitial tumor ablation (RITA) of the prostate. Tech Urol 4: 103-109, 1998.

21. Hoey MF, Dixon CM and Paul S: Transurethral prostate ablation using saline-liquid electrode introduced via flexible cystoscope. J Endourol 12: 461-468, 1998.

22. Foster RS, Bihrle R, Sanghvi N, Fry F, Kopecky K, Regan J, Eble J, Hennige C, Hennige LV and Donohue JP: Production of prostatic lesions in canines using transrectally administered high-intensity focused ultrasound. Eur Urol 23: 330-336, 1993.

23. Peters RD, Chan E, Trachtenberg J, Jothy S, Kapusta L, Kucharczyk W and Henkelman RM: Magnetic resonance thermometry for predicting thermal damage: An application of interstitial laser coagulation in an in vivo canine prostate model. Magn Reson Med 44: 873-883, 2000. 